\title{
ON THE CONVERGENCE OF AN ITERATIVE METHOD FOR THE MINIMAX PROBLEM
}

\author{
WENYU SUN ${ }^{1}$
}

(Received 17 November 1995; revised 19 March 1996)

\begin{abstract}
In this paper we present an Extended Linear-Quadratic Programming method for the minimax problem. We show that the Extended Linear-Quadratic Programming method for the minimax problem is equivalent to the Josephy-Newton method for generalized equation, and establish the local convergence result. Furthermore, we obtain the global convergence result for the minimax problem by means of the equivalence relation between the generalized equation and the normal equation.
\end{abstract}

\section{Introduction}

In this paper we consider the minimax problem

$$
\min _{x \in X} \max _{y \in Y} L(x, y),
$$

where $L(x, y)$ is a saddle function, that is, $L$ is a convex-concave function from $X \times Y \rightarrow[-\infty,+\infty]$, that is, $L(\cdot, y)$ is a convex function on $X$ for each $y \in Y$ and $L(x, \cdot)$ a concave function on $Y$ for each $x \in X$, where $X$ and $Y$ are closed nonempty convex subsets in $R^{n}$ and $R^{m}$, respectively.

Many minimax problems often arise in engineering design [9], computer-aideddesign [10], circuit design [23], chemical design and optimal control [18, 19] and others.

At present, there are several existing methods for the minimax problem (1), for example, the feasible direction method [6], the projected Lagrange method [5], a combined Linear Programming and quasi-Newton method and the barrier function method. In [13] we research the Kuhn-Tucker condition for the minimax problem (1), and discuss the local convergence using the Kuhn-Tucker equation and the Sequential Quadratic Programming method for the dual-pair problem.

${ }^{1}$ Department of Mathematics, Nanjing University, Nanjing 210093, China

(C) Australian Mathematical Society, 1997, Serial-fee code 0334-2700/97 
In this paper we transform the minimax problem into a sequence of Extended Linear-Quadratic Programming (ELQP) problems which are introduced by Rockafellar and Wets [20] in stochastic programming and which can be effectively solved by some existing algorithms. In addition, by means of some transformations, we can also use the existing effective algorithms for linear complementarity or linear variational inequality to get solutions of ELQP problems, which converge to the solutions of the original minimax problem (1). In order to establish the convergence results, we discuss how to transform the ELQP subproblem into the model problem of the Josephy-Newton method and Robinson-Newton method, respectively, and then establish the local and global convergence results. Our approach provides a new way to prove convergence results for minimax problems.

The organization of the remainder of this paper is as follows. Section 2 establishes the ELQP problem as a subproblem of the minimax problem. Section 3 gives local convergence result by using a result of the Josephy-Newton method and Section 4 discusses the global convergence via the Robinson-Newton method.

\section{ELQP problem as a subproblem}

Consider the minimax problem (1). From Lemma 36.1 of [18], we have

$$
\max _{y \in Y} \min _{x \in X} L(x, y) \leq \min _{x \in X} \max _{y \in Y} L(x, y) .
$$

Therefore, a point $(\bar{x}, \bar{y})$ is a saddle point of $L$ if and only if the minimum of

$$
\min _{x \in X}\left\{\max _{y \in Y} L(x, y)\right\}
$$

is attained at $\bar{x}$, and the maximum of

$$
\max _{y \in Y}\left\{\min _{x \in X} L(x, y)\right\}
$$

is attained at $\bar{y}$, and these two extremes are equal, that is,

$$
\max _{y \in Y}\left\{\min _{x \in X} L(x, y)\right\}=\min _{x \in X}\left\{\max _{y \in Y} L(x, y)\right\}
$$

or

$$
L(\bar{x}, \bar{y})=\min _{x \in X} L(x, \bar{y})=\max _{y \in Y} L(\bar{x}, y) .
$$

Equations (3) and (4) can also be written as

$$
\begin{aligned}
& (\mathscr{P}) \min _{x \in X} L(x, \bar{y}), \\
& (\mathscr{D}) \quad \max _{y \in Y} L(\bar{x}, y) .
\end{aligned}
$$


The saddle point condition

$$
L(\bar{x}, y) \leq L(\bar{x}, \bar{y}) \leq L(x, \bar{y}), \quad \forall x \in X, y \in Y
$$

holds if and only if the convex function $L(\cdot, \bar{y})$ achieves its minimum at $\bar{x}$, that is,

$$
0 \in \partial_{x} L(\bar{x}, \bar{y})+N_{X}(\bar{x}),
$$

and the concave function $L(\bar{x}, \cdot)$ achieves its maximum at $\bar{y}$, that is,

$$
0 \in \partial_{y} L(\bar{x}, \bar{y})+N_{Y}(\bar{y}),
$$

where $\partial_{x} L(\bar{x}, \bar{y})$ and $\partial_{y} L(\bar{x}, \bar{y})$ denote the convex and concave subdifferentials of $L$ at $(\bar{x}, \bar{y})$ about $x$ and $y$ respectively, and $N_{X}(\bar{x})$ and $N_{Y}(\bar{y})$ denote normal cones of $X$ and $Y$ at $\bar{x}$ and $\bar{y}$ respectively. Therefore the saddle point condition (9) is equivalent to

$$
(0,0) \in \partial L(\bar{x}, \bar{y})+N_{X \times Y}(\bar{x}, \bar{y}),
$$

where $\partial L(\bar{x}, \bar{y})$ denotes the saddle function subdifferential of $L$ at $(\bar{x}, \bar{y})$ and $N_{X \times Y}(\bar{x}$, $\bar{y})$ denotes the normal cone of $X \times Y$ at $(\bar{x}, \bar{y})$. If $X=R^{n}, Y=R^{m}$ and $L$ is a continuously differentiable convex-concave function on $R^{n} \times R^{m}$, then (12) can be reduced to

$$
\nabla L(\bar{x}, \bar{y})=\left(\begin{array}{l}
\nabla_{x} L(\bar{x}, \bar{y}) \\
\nabla_{y} L(\bar{x}, \bar{y})
\end{array}\right)=0
$$

which can be solved by Newton's method.

For the constrained minimax problem (1) with continuous function $L$, its equivalent problem-pair is

$$
\begin{aligned}
& \min _{x \in X} L(x, \bar{y}), \\
& \max _{y \in Y} L(\bar{x}, y),
\end{aligned}
$$

where

$$
\bar{y}=\arg \max _{y \in Y} L(x, y), \quad \bar{x}=\arg \min _{x \in X} L(x, y) .
$$

Then the solution $(\bar{x}, \bar{y})$ of the problem (1) satisfies

$$
\begin{aligned}
& 0 \in \nabla_{x} L(x, \bar{y})+N_{X}(x), \\
& 0 \in-\nabla_{y} L(\bar{x}, y)+N_{Y}(y),
\end{aligned}
$$


which means the solution $\bar{z}$ of (1) satisfies the necessary optimality condition

$$
0 \in-\nabla L(z)+N_{Z}(z),
$$

where $z=(x, y), \nabla L(z)=\left(-\nabla_{x} L(x, y), \nabla_{y} L(x, y)\right), Z=X \times Y, N_{Z}=N_{X} \times N_{Y}$.

In order to find the saddle point of the minimax problem (1), we construct a sequence $\left\{\left(x_{k}, y_{k}\right)\right\}$ of $(n+m)$-vectors which is an estimate of the problem (1). We first linearize $\nabla L$ to get a series of Extended Linear-Quadratic Programming (ELQP) subproblems, then solve these ELQP subproblems to obtain the solution sequence $\left\{\left(x_{k}, y_{k}\right)\right\}$. This approach is similar to the SQP the (Sequential Quadratic Programming) method for the optimization problem (see $[12,21,22]$ ). In the following two sections, we prove that this solution sequence $\left\{\left(x_{k}, y_{k}\right)\right\}$ converges to the solution $(\bar{x}, \bar{y})$ of the minimax problem locally and globally. These ELQP subproblems are introduced by Rockafellar and Wets [20] in stochastic programming, and can be solved by some effective algorithms, for example, primal-dual projected gradient method, primaldual conjugate gradient method, and sequential quadratic programming method (see $[18,19,24])$. In this paper we restrict our attention to the motivation and convergence of an iterative method for the minimax problem (1), and do not discuss the algorithm's details and modifications for the ELQP subproblem.

We now suppose $L$ is a twice continuously differentiable function on $X \times Y$. We consider an extended linear-quadratic programming subproblem

$$
\min _{x \in X} \max _{y \in Y} L_{k}(x, y)
$$

for (1) with

$$
\begin{aligned}
L_{k}(x, y)= & \nabla_{x} L\left(x_{k}, y_{k}\right)^{T}\left(x-x_{k}\right)+\nabla_{y} L\left(x_{k}, y_{k}\right)^{T}\left(y-y_{k}\right) \\
& +\frac{1}{2}\left(x-x_{k}\right)^{T} P_{k}\left(x-x_{k}\right)-\frac{1}{2}\left(y-y_{k}\right)^{T} Q_{k}\left(y-y_{k}\right) \\
& -\left(y-y_{k}\right)^{T} R_{k}\left(x-x_{k}\right),
\end{aligned}
$$

where $P_{k} \in R^{n \times n}, Q_{k} \in R^{m \times m}$, and $R_{k} \in R^{m \times n}$ are the second order partial derivative matrices of the function $L$ or their approximations. We assume that $P_{k}$ and $Q_{k}$ are positive definite matrices.

If let $z=(x, y)$ and

$$
G\left(z_{k}\right)=\left[\begin{array}{cc}
P_{k} & -R_{k}^{T} \\
-R_{k} & -Q_{k}
\end{array}\right]
$$

where $G\left(z_{k}\right)$ is $\nabla^{2} L\left(x_{k}, y_{k}\right)$ or its approximation, then the function (17) can be written as

$$
L_{k}(z)=\nabla L\left(z_{k}\right)^{T}\left(z-z_{k}\right)+\frac{1}{2}\left(z-z_{k}\right)^{T} G\left(z_{k}\right)\left(z-z_{k}\right)
$$


This shows that $L_{k}(z)$ is a linearization of $\nabla L(z)$ at $z_{k}$, and also implies that the rationale behind employing the ELQP subproblems in the minimax problem is that the ELQP problem is similar to the quadratic programming subproblem in the SQP method for solving constrained optimization.

For the unconstrained minimax problem, that is, $X=R^{n}$ and $Y=R^{m}$, the point $\left(x_{k}^{*}, y_{k}^{*}\right)$ is a saddle point of (16) if and only if $\left(x_{k}^{*}, y_{k}^{*}\right)$ satisfies the Newton's iteration for (13). For constrained minimax problem, the point $\left(x_{k}^{*}, y_{k}^{*}\right)$ is a saddle point of (16) if and only if $\left(x_{k}^{*}, y_{k}^{*}\right)$ satisfies the Newton's iteration for (12).

We can also use some methods for linear variational inequality or linear complementarity to deal with ELQP subproblem (16). In fact, from Theorem 2.3 in [19], the saddle point optimality condition can be written as the linear variational inequality

$$
\begin{aligned}
-\nabla_{x} L\left(x_{k}^{*}, y_{k}^{*}\right)\left(x-x_{k}^{*}\right) & \leq 0, \\
\nabla_{y} L\left(x_{k}^{*}, y_{k}^{*}\right)\left(y-y_{k}^{*}\right) & \leq 0 .
\end{aligned}
$$

The above variational inequality can also be transformed to a linear complementarity problem.

\section{Local convergence}

In this section we shall establish the local convergence of our iterative algorithm. First we state the algorithm as follows.

AlgORITHM 1. STEP 1: Start with an estimate $\left(x_{0}, y_{0}\right)$ of a saddle point of problem (1). Set $k=0$.

STEP 2: Having $\left(x_{k}, y_{k}\right)$, find a saddle point $\left(x_{k+1}, y_{k+1}\right)$ of the extended linearquadratic programming subproblem (16).

STEP 3: If $\left(x_{k+1}, y_{k+1}\right)$ satisfies a prescribed convergence criterion, stop; otherwise set $k:=k+1$ and go to Step 2 .

Before proving the formal theorem, we give two definitions.

DEFINITION 1. Let $Z \in R^{n} \times R^{n}$ be a closed, convex, nonempty set. The normal cone to $Z$ at $a$ is

$$
N_{Z}(a):=\left\{w \mid \quad w^{T}(z-a) \leq 0, \forall z \in Z\right\}
$$

DEFINITION 2 (Robinson [16]). Consider the generalized equation

$$
0 \in F(z)+N_{Z}(z)
$$


where $F: R^{n} \times R^{m} \rightarrow R^{n} \times R^{m}$ is Frechet differentiable at $z_{0}$ which is a solution of (21). Define the operator $T$ as

$$
T(z):=F\left(z_{0}\right)+F^{\prime}\left(z_{0}\right)\left(z-z_{0}\right)+N_{Z}(z)
$$

If there is a neighborhood $\Omega_{0}$ of 0 and a neighborhood $\Omega_{z_{0}}$ of $z_{0}$, such that $\left(T^{-1} \cap\right.$ $\left.\Omega_{z_{0}}\right) \mid \Omega_{0}$ is single valued and Lipschitzian, that is, for each $\omega \in \Omega_{0}$, there is a set of $\tau \in \Omega_{z_{0}}$ such that $\omega \in T(\tau)$, and the function is Lipschitzian, then $z_{0}$ is called a regular solution of (21).

Robinson $[15,16]$ first established mathematical foundations for generalized equations, researched their regularity and solutions. Following Robinson's idea, Josephy gives a convergence theorem of Newton's method for a generalized equation which is a generalization of a classical result taken from Ortega and Rheinboldt [7]. Newton's method for solving the nonlinear equation $0=F(z)$ consists of replacing $F$ by its linearization $L F_{z_{0}}(z)=F\left(z_{0}\right)+F^{\prime}\left(z_{0}\right)\left(z-z_{0}\right)$ and solving $0=L F_{z_{0}}(z)$. The Josephy-Newton method for solving a generalized equation $0 \in F(z)+N_{Z}(z)$ consists of replacing $F$ by $L F_{z_{0}}(z)$ and solving the linearized generalized equation $0 \in L F_{z 0}(z)+N_{Z}(z)$. In the following, we establish the local convergence theorem of Algorithm 1. In this theorem we use Josephy's result [2] to prove that the solution sequence of the ELQP subproblem converges to the solution of minimax problem (1). Note that in the following theorem $B(z, \rho)$ denotes a closed ball with center $z$ and radius $\rho$.

THEOREM 1. Let $L: Z \subset R^{n} \times R^{m} \rightarrow R$ be second order continuously differentiable, $\nabla L$ have Lipschitz continuous Frechet derivative on $Z, l$ be Lipschitz modulus. Let $z_{0} \in Z$. Suppose that the generalized equation

$$
0 \in-\nabla L\left(z_{0}\right)-\nabla^{2} L\left(z_{0}\right)\left(z-z_{0}\right)+N_{Z}(z)
$$

has regular solution $z_{1}$, and the corresponding Lipschitz modulus is $l_{1}$. Choose $r_{1}>0, r_{2}>0$ and $\rho>0$, such that for any $z \in B\left(z_{0}, \rho\right)$,

$$
\left[\nabla L(z)+\nabla^{2} L(z)^{T}((\cdot)-z)+N_{Z}(\cdot)\right]^{-1} \cap B\left(z_{1}, r_{1}\right)
$$

is single-valued and Lipschitzian with Lipschitz modulus $l_{1}\left[1-l_{1} \| \nabla^{2} L(z)-\right.$ $\left.\nabla^{2} L\left(z_{1}\right) \|\right]^{-1}$ when it is restricted on $B\left(0, r_{2}\right)$.

Define

$$
\eta:=\left\|z_{1}-z_{0}\right\|, \quad h:=l_{1} \eta
$$

Assume that

(a) $0<h \leq 1 / 2$, 
(b) $l \eta^{2} \leq 2 r_{2}$,

(c) $B\left(z_{0}, t^{*}\right) \subset Z \cap B\left(z_{0}, \rho\right)$,

where

$$
t^{*}=\left[\frac{1-(1-2 h)^{1 / 2}}{h}\right] \eta .
$$

Then the sequence $\left\{z_{k}\right\}$ generated by solving the ELQP subproblem is well-defined, and converges to $\bar{z} \in B\left(z_{0}, t^{*}\right)$ which is the solution of minimax problem (1). Furthermore, for each $k \geq 1$, we have

$$
\left\|\bar{z}-z_{k}\right\| \leq\left(z^{n+m} l l_{1}\right)^{-1}(2 h)^{2^{k}}
$$

PROOF. Let $(\bar{x}, \bar{y})$ be the solution of (1). Note that $(15)$ is equivalent to

$$
-\nabla_{x} L(\bar{x}, \bar{y}) \in N_{X}(\bar{x}), \quad \nabla_{y} L(\bar{x}, \bar{y}) \in N_{Y}(\bar{y})
$$

which implies that, by the definition of normal cone,

$$
\begin{aligned}
-\nabla_{x} L(\bar{x}, \bar{y})^{T}(x-\bar{x}) & \leq 0, \quad \forall x \in X, \\
\nabla_{y} L(\bar{x}, \bar{y})^{T}(y-\bar{y}) & \leq 0, \quad \forall y \in Y .
\end{aligned}
$$

Set $z=(x, y)$ and

$$
\nabla L(z)=\left[\begin{array}{c}
-\nabla_{x} L(z) \\
\nabla_{y} L(z)
\end{array}\right]
$$

Then (24) is

$$
\nabla L(\bar{z}) \in N_{Z}(\bar{z})
$$

which means that

$$
\nabla L(\bar{z})^{T}(z-\bar{z}) \leq 0, \quad \forall z \in Z
$$

where $Z=X \times Y, N_{Z}(\bar{z})=N_{X}(\bar{x}) \times N_{Y}(\bar{y})$. Assume $F(z)=-\nabla L(z)$. It follows from (26) that

$$
0 \in F(\bar{z})+N_{Z}(\bar{z})
$$

which implies that in order to get the solution $\bar{z}$ of (1), we require to solve the generalized equation

$$
0 \in F(z)+N_{Z}(z)
$$


Similarly, we consider ELQP subproblem (16). Let $\left(x_{k+1}, y_{k+1}\right)$ be the solution of (16). Then we have

$$
\begin{cases}-\nabla_{x} L_{k}\left(x_{k+1}, y_{k+1}\right)\left(x-x_{k+1}\right) \leq 0, & \forall x \in X, \\ \nabla_{y} L_{k}\left(x_{k+1}, y_{k+1}\right)\left(y-y_{k+1}\right) \leq 0, & \forall y \in Y .\end{cases}
$$

Set

$$
\nabla L_{k}(z)=\left[\begin{array}{c}
-\nabla_{x} L_{k}(z) \\
\nabla_{y} L_{k}(z)
\end{array}\right]
$$

Then

$$
\nabla L_{k}\left(z_{k+1}\right)^{T}\left(z-z_{k+1}\right) \leq 0, \quad \forall z \in Z,
$$

that is,

$$
0 \in-\nabla L_{k}\left(z_{k+1}\right)+N_{Z}\left(z_{k+1}\right)
$$

Since

$$
\nabla L_{k}(z)=\nabla L\left(z_{k}\right)+G\left(z_{k}\right)\left(z-z_{k}\right),
$$

then, from (19), the solution sequence $\left\{z_{k+1}\right\}$ satisfies

$$
\begin{aligned}
0 & \in-\nabla L\left(z_{k}\right)-G\left(z_{k}\right)\left(z-z_{k}\right)+N_{Z}(z) \\
& =F\left(z_{k}\right)+\nabla F\left(z_{k}\right)\left(z-z_{k}\right)+N_{Z}(z),
\end{aligned}
$$

which is just a linearization of the generalized equation (29). From the assumptions of the theorem, the generalized equation $0 \in T_{z_{0}}(z)$ is regular at $z_{1}$ and

$$
z_{n+1}=T_{z_{n}}^{-1}(0) \cap B\left(z_{1}, r_{1}\right)
$$

is well-defined. From Josephy's theorem [2, Theorem 2], we obtain that the solution sequence $\left\{z_{k+1}\right\}$ of (32) converges to the solution $\bar{z} \in B\left(z_{0}, t^{*}\right)$ of (29), and therefore converges to the solution of minimax problem (1).

Furthermore, from Josephy theorem [2] again, we get

$$
\left\|\bar{z}-z_{k}\right\| \leq\left(2^{n+m} l l_{1}\right)^{-1}(2 h)^{2^{k}} .
$$

The proof is completed.

REMARK. Similarly, if $G\left(z_{k}\right)$ is some approximation to $\nabla^{2} L\left(z_{k}\right)$ which satisfies some suitable conditions, then the superlinear convergence of the quasi-Newton method for minimax problem (1) can be established from another Josephy theorem [3]. If $h<1 / 2$ in Theorem 1, then (23) yields R-quadratic convergence. 


\section{Global convergence}

The above discussion indicates that the ELQP method for minimax problem (1) is equivalent to the Josephy-Newton method

$$
0 \in F\left(z_{k}\right)+\nabla F\left(z_{k}\right)\left(z-z_{k}\right)+N_{Z}(z)
$$

for generalized equation $0 \in F(z)+N_{z}(z)$, where $F(z)=-\nabla L(z)$, and establishes the local convergence. In this section, following Ralph's global convergence for nonsmooth equations [14], we show the global convergence of our method.

Consider the normal equation $F_{Z}(w)=0$, where $w \in R^{n+m}$,

$$
F_{Z}(w)=F(\pi(w))+w-\pi(w)=F(z)+w-z,
$$

which is defined and discussed by Robinson [17], where $z=\pi(w)$ and $\pi(\cdot)$ is a projector. Construct a first-order approximation of $F_{Z}$

$$
A_{k}(w)=F\left(z_{k}\right)+\nabla F\left(z_{k}\right)\left(z-z_{k}\right)+w-z,
$$

so the next Newton's iterate is $\hat{w}_{k+1}=A_{k}^{-1}(0)$. Consider the Newton's path

$$
p_{k}(t)=w_{k}+t\left(\hat{w}_{k+1}-w_{k}\right)
$$

which is such that

$$
A_{k}\left(p_{k}(t)\right)=(1-t) F_{Z}\left(w_{k}\right)
$$

Hence we have

$$
F_{Z}\left(p_{k}(t)\right)=(1-t) F_{Z}\left(w_{k}\right)+o(t)
$$

Choose $\sigma \in(0,1)$. The monotone path search rule is to find $t_{k} \in\left[0, T_{k}\right], T_{k} \in[0,1]$, such that $t_{k}$ satisfies

$$
\left\|F_{Z}\left(p_{k}(t)\right)\right\|<(1-\sigma t)\left\|F_{Z}\left(w_{k}\right)\right\|,
$$

and then set $w_{k+1}=p_{k}(t)$.

REMARK 1. In search rule (36), we can use the exact search rule or the inexact search rule. 
REMARK 2. Instead of the monotone search rule (36), we may also use the nonmonotone path search rule which is presented by Grippo et al. [1]. In this case (36) is changed to

$$
\left\|F_{Z}\left(p_{k}(t)\right)\right\|<(1-\sigma t) \max \left\{\left\|F_{Z}\left(w_{k+1-j}\right)\right\| \| \quad j=1, \cdots, \min \{M, k+1\}\right\},
$$

where the positive integer $M$ is a control parameter. When $M=1,(37)$ is just (36).

REMARK 3. Instead of the path search, we may use trust region strategy to obtain global convergence. For a detailed discussion, see Powell [11], Moré [4] and Sun and Yuan [22].

DEFINITION 3. The second-order sufficiency condition of the minimax problem (1) is

$$
\begin{array}{lll}
d_{1}^{T} \nabla_{x}^{2} L d_{1}>0, & \forall d_{1} \neq 0, & d_{1} \in T_{X}(x), \\
d_{2}^{T} \nabla_{y}^{2} L d_{2}<0, & \forall d_{2} \neq 0, & d_{2} \in T_{Y}(y),
\end{array}
$$

where $T_{X}(x)$ and $T_{Y}(y)$ are tangent cone to $X$ and $Y$ at $x$ and $y$ respectively.

Ralph [14] uses the following assumptions in the convergence. Let $f: X \rightarrow Y$ be continuous, $\alpha_{0}>0$ and $X_{0}=\left\{x \in X \mid\|f(x)\| \leq \alpha_{0}\right\}$. Assume

A1. $\mathscr{A}$ is a uniform first-order approximation of $f$ on $X_{0}$.

A2. $\mathscr{A}$ is uniformly Lipschitzianly invertible near each $x \in X_{0}$.

A3. There exist paths used by the algorithm.

Under the above assumptions, Ralph [14] proves that for any $x_{0} \in X_{0}$, path search damped Newton's method is well defined and the sequence $\left\{x_{k}\right\}$ converges to zero $x^{*}$ of $f$.

Below we present the global convergence theorem.

THEOREM 2. Consider the minimax problem (1). Let $Z$ be a closed, convex, nonempty set in $R^{n} \times R^{m}$ and $L: Z \rightarrow R$ twice continuously differentiable. Assume the second order sufficiency condition (38) holds, and the level set

$$
Z_{0}=\left\{z \in R^{n} \times R^{m} \mid \quad\left\|F_{Z}(z)\right\| \leq \alpha_{0}, \alpha_{0}>0\right\}
$$

is bounded. Then, for any $z_{0} \in Z_{0}$, the sequence $\left\{z_{k}\right\}$ generated by the ELQP method with path search is well-defined and converges to the solution $\bar{z}$ of the minimax problem (1).

PROOF. It is well known from the proof of the local convergence that solving problem (1) is equivalent to solving (7) and (8), which is also equivalent to solving

$$
0 \in-\nabla L(z)+N_{Z}(z) \text {. }
$$


Set $F(z)=-\nabla L(z)$. Then $F$ is continuously differentiable and

$$
0 \in F(z)+N_{Z}(z)
$$

Consider

$$
F\left(\pi_{Z}(w)\right)+w-\pi_{Z}(w)=0,
$$

where $\pi_{Z}(w)$ denotes the nearest point in $Z$ to $w$ with respect to the Euclidean norm. Setting

$$
F_{Z}(w)=F\left(\pi_{Z}(w)\right)+w-\pi_{Z}(w)
$$

we have

$$
F_{Z}(w)=0, \quad w \in R^{n+m} .
$$

According to Robinson [17], if $w$ solves (41), then $z=\pi_{Z}(w)$ solves (39). Furthermore, $z$ solves the minimax problem (1).

Next we consider the ELQP method with path search for (1)

$$
\min _{x \in X} \max _{y \in Y} L_{k}(x, y),
$$

where $L_{k}$ is defined by (17). It follows from (31) and (32) that this method is equivalent to the Josephy-Newton method

$$
0 \in F\left(z_{k}\right)+\nabla F\left(z_{k}\right)\left(z-z_{k}\right)+N_{Z}(z)
$$

for (39). The Robinson-Newton method for the normal equation is

$$
A_{k}(w)=F\left(z_{k}\right)+\nabla F\left(z_{k}\right)\left(z-z_{k}\right)+w-z=0
$$

and

$$
\hat{w}_{k+1}=\hat{z}_{k+1}-F\left(z_{k}\right)-\nabla F\left(z_{k}\right)\left(\hat{z}_{k+1}-z_{k}\right),
$$

where $z_{k}=\pi_{Z}\left(w_{k}\right), \hat{z}_{k+1}=\pi_{Z}\left(\hat{w}_{k+1}\right)$. The path search on $\left[w_{k}, \hat{w}_{k+1}\right]$ produces $w_{k+1}$ and the projection $z_{k+1}=\pi_{Z}\left(w_{k+1}\right)$. As $L(z)$ is twice continuously differentiable, the second-order sufficiency condition for minimax problem (1) holds, and $\nabla L(z)_{Z}$ is locally Lipschitzianly invertible from Lemma 14 of [14]. So the second-order approximation $L_{k}(z)$ of $L(z)$ is uniformly continuous and Lipschitzianly invertible. Note that $A_{k}(w)$ is a first order approximation of $F_{Z}$ which satisfies condition Al and $A 2$ and the path search satisfies (35) which means condition A3. Therefore, we obtain directly that, by means of Theorem 9 of [14], for any $w_{0} \in Z_{0}$, the sequence 
$\left\{w_{k}\right\}$ generated from Robinson-Newton method converges to the solution $\bar{w}$ of the normal equation (41). Equivalently, this shows that the sequence $\left\{z_{k}\right\}$ generated by the Josephy-Newton method converges to the solution $\bar{z}=\pi_{Z}(\bar{w})$ of the generalized equation (39), that is, the sequence $\left\{z_{k}\right\}$ generated by the ELQP method converges to the solution of (1). Furthermore, it follows from Theorem 9 of [14] again that the convergence rate is Q-superlinear.

REMARK. Similarly, we can also use the B-differentiability concept of Pang [8], that is, use

$$
B_{k}(w)=F_{Z}\left(w_{k}\right)+F_{Z}^{\prime}\left(w_{k}, w-w_{k}\right),
$$

as a first-order approximation of $F_{Z}(w)$ instead of $A_{k}(w)$ in (33), and prove the global convergence result of B-differentiable Newton's method with line search.

\section{Acknowledgement}

The author thanks Prof. Liqun Qi for helpful discussion when the author visited the University of New South Wales, Australia. The author also thanks Dr. Daniel Ralph for his encouragement and providing his manuscript. This work was supported by the National Natural Science Foundation of China and CNPq, Brazil.

\section{References}

[1] L. Grippo, F. Lampariello and S. Lucidi, "A nonmonotone line search technique for Newton's method", SIAM Journal of Numerical Analysis 23 (1986) 707-716.

[2] N. H. Josephy, "Newton's method for generalized equations", National Technical Information Service, (Springfield, VA 22161, USA. Accession number AD A077 096).

[3] N.H. Josephy, "Quasi-newton methods for generalized equations", National Technical Information Service, (Springfield, VA 22161, USA. Accession number AD A077 096).

[4] J. J. Moré, "Recent developments in algorithms and software for trust region methods", in Mathematical Programming, The State of Art, Bonn 1982 (eds. A. Bachem, M. Grötschel and B. Korte), (Springer-Verlag, Berlin, 1983) 258-287.

[5] W. Murray and M. L. Overton, "A projected Langrangian algorithm for nonlinear minimax optimization", SIAM J. Sci. Stat. Comp. 1 (1980) 345-370.

[6] W. Oettli, "The method of feasible directions for continuous minimax problems", in Survey in Mathematical Programming (ed. A. Prekopa), Volume 1, (North-Holland, Amsterdam, 1979) $505-512$.

[7] J. M. Ortega and W. C. Rheinboldt, Iterative Solution of nonlinear equations of several variables (Academic Press, San Diego, 1970).

[8] J. S. Pang, "Newton's method for B-differentiable equations", Mathematics of Operations Research 15 (1990) 311-341. 
[9] E. Polak, "On the mathematical foundations of nondifferentiable optimization", SIAM Review 29 (1987) 21-87.

[10] E. Polak, J. E. Higgins and D. Q. Mayne, "A barrier function method for minimax problems", Mathematical Programming 54 (1992) 155-176.

[11] M. J. D. Powell, "Convergence properties of a class of minimization algorithm", in Nonlinear Programming 2 (eds. O. L. Mangasarian, R. R. Meyer and S. M. Robonson), (Academic Press, New York, 1975).

[12] M. J. D. Powell, "Variable metric methods for constrained optimization", in Mathematical Programming, The State of Art, Bonn 1982 (eds. A. Bachem, M. Grötschel and B. Korte), (SpringerVerlag, Berlin, 1983) 288-311.

[13] L. Qi and W. Sun, "An iterative method for the minimax problem", in Minimax and Applications (eds. D. Z. Du and P. M. Pardalos), (Kulwer Academic Publisher, Boston, 1995) 55-67.

[14] D. Ralph, "Global convergence of damped Newton's method for nonsmooth equations, via the path search", Mathematics of Operations Research 19 (1994) 352-389.

[15] S. M. Robinson, "Generalized equation and their solutions, Part I: Basic theory", Mathematical Programming Study 10 (1979) 128-141.

[16] S. M. Robinson, "Strongly regular generalized equations", Mathematics of Operations Research 5 (1980) 43-62.

[17] S. M. Robinson, "Normal maps induced by linear transformations", Mathematics of Operations Research 17 (1992) 691-714.

[18] R. T. Rockafellar, "Linear-quadratic programming and optimal control", SIAM Journal on Control and Optimization 25 (1987) 781-814.

[19] R. T. Rockafellar, "Computational schemes for large-scale problems in extended linear-quadratic programming", Mathematical Programming 48 (1990) 447-474.

[20] R. T. Rockafellar and R. J.-B. Wets, "A dual solution procedure for quadratic stochastic programs with simple recourse", in Numerical Methods, Lecture Notes in Mathematics 1005 (ed. A. Reinoza), (Springer-Verlag, Berlin, 1983) 252-265.

[21] W. Sun, "Newton's method and quasi-Newton-SQP method for general constrained optimization", to appear in Applied Mathematics and Computation (1997).

[22] W. Sun and Y. Yuan, Optimization Theory and Methods (Science Press, Beijing, 1997).

[23] S. E. Sussman-Fort, "Approximate direct-search minimax circuit optimization", International J. for Numerical Methods in Engineering 28 (1989) 751-783.

[24] C. Zhu and R. T. Rockafellar, "Primal-dual projected gradient algorithms for extended linear quadratic programming", SIAM J. Optimization 3 (1993) 751-783. 